\title{
CONSTRUCCIÓN Y VALIDACIÓN DE UN INSTRUMENTO PARA LA MEDICIÓN DE DIMENSIONES SOCIALES EN LAS PRÁCTICAS DEPORTIVAS DE UNA SUBCULTURA DEPORTIVA ESPECÍFICA: EL TRIATLÓN DE LARGA DISTANCIA
}

\author{
CONSTRUCTION AND VALIDATION OF AN INSTRUMENT FOR \\ THE MEASUREMENT OF SOCIAL DIMENSIONS IN THE SPORTS \\ PRACTICES OF A SPECIFIC SPORT SUBCULTURE: THE LONG \\ DISTANCE TRIATHLON
}

Eloy García García Universidad de Alicante, España eloy.garcia@ua.es

\begin{abstract}
Resumen
A través de este trabajo se describe el proceso de construcción, así como el testado en términos de validez y de fiabilidad del instrumento utilizado en una investigación aun en curso que pretende identificar las principales características sociales, introduciendo la perspectiva de género en una subcultura específica de creciente interés dentro del escenario deportivo actual, el triatlón de larga distancia. Para ello se enumeran los pasos seguidos en la integración metodológica cualitativa-cuantitativa que se ha llevado a cabo para configurar las variables empíricas definitivas del cuestionario, se describen las temáticas generales que se tratan en el cuestionario y los test realizados para establecer la validez y fiabilidad del instrumento que se utilizará con carácter exploratorio. Las escalas utilizadas se resumen en 5 factores que explican un $72,61 \%$ de la varianza donde la medida $\mathrm{KMO}$ es 0,51 , y la prueba de esfericidad
\end{abstract}


Eloy García García

de Bartlett presenta Sig=,000). En cuanto a la fiabilidad, alfa de Cronbach ofrece resultados en torno a 0,5 o superiores en los factores y subescalas que los componen, demostrando fiabilidad con finalidad exploratoria en escalas que tienen menos de 10 items.

Palabras clave: Instrumento cuantitativo, validez, fiabilidad, triatlón de larga distancia, género.

\begin{abstract}
In this paper we want to describe the construction process and also the validity and reliability of our sociological investigation method. Such a method will be applied in our ongoing research project. The main objective of this research is to describe the way of life of long distance runners and also those enrolled in half and full ironman triathlon races. Moreover, it is also studied how gender may affect results. To do this, we list the steps followed in a qualitative-quantitative methodological integration that has been carried out to configure the empirical variables for the questionnaire, describe the general topics that are addressed in the questionnaire and the tests performed to establish the validity and reliability of the instrument to be used on an exploratory basis. The scales used are summarized in 5 factors that explain $72.61 \%$ of the variance where the KMO measure is 0.51, and the Bartlett sphericity test has Sig =,000). As for reliability, Cronbach's alpha statistic offers results around 0.5 or higher in the factors and subscales that compose them, demonstrating reliability with an exploratory purpose in scales that have less than 10 items.
\end{abstract}

Keywords: quantitative instrument, validity, reliability, long distance triathlon

\title{
Extended abstract
}

In this paper we want to describe the construction process and also the validity and reliability of our sociological investigation method. Such a method will be applied in our ongoing research project.

In the last two decades there was an increasing interest in long distance races and, in particular, triathlon has emerged as a popular middle class sport. In Spain, the last five years the number of licenses has increased over $64 \%$ (see Table 2). The main objective of this research is to describe the way of life of long distance runners and also those enrolled in half and full ironman triathlon races. Moreover, it is also studied how gender may affect results.

To reach these objectives we employ a sociological mixture methodology assuming the complementarity between different perspectives. We combine different quantitative and qualitative perspectives aimed to design a research survey as main tool. First, a qualitative methodology was implemented. Taking 
a subset of well-informed subjects (those who took part in many long distance races) we conduct in-depth interviews to detect the main general concepts, develop questions, and establish categories of subjective variables.

Once the structure of the questionnaire was done, we run a training test in order to skip irrelevant and/or redundant questions getting a final document comprising 69 questions split in 7 themes. It is expected a running time of 15' per subject.

The research survey was applied to subject that took part in two long distance Spanish Cup races. Once the athletes were listed in the race we sent emails. The sample was extracted from a sampling frame composed of a list of the emails of the triathlete's registered in the two races. After depuration of duplicities and irregularities the total was 1800 subjects.

With our frame sample framework we proceeded with a non-probabilistic sampling derived from the type of self-administered interview via email. In order to calculate the sample size and also the error, the probabilistic rules of simple random sampling for finite populations were followed. The final sample size was 270 cases. After debugging and testing, the number of cases accepted for the analysis was 230, which coincides with the subjects who completed all the variables that correspond to the objectives and hypotheses raised.

The choice of internet for the distribution of questionnaires was motivated by the advantages provided by the Internet. Indeed, it is possible to deliver a huge number of questionnaires at a lower cost and, in addition, with space-time flexibility to respond. This type of survey presents difficulties in coverage, the bias in variables such as age, educational level and the low response rate. None of us could estimate the level of Internet coverage, but we did add questionnaire questions so that it could be filled by using different devices: tablet, mobile phones as well as the classic computer format. In addition, with the results obtained ex-post, it can be said that the profile of the long distance triathlete is a medium high socioeconomic level, which correlates directly with the internet access and level of coverage. Regarding the low response rate of this type of survey could be smoothed through incentive mode of draw of 12 prizes that were delivered at the end of the period of completion. Response rate was $12.8 \%$.

Validity of the model means the congruence or goodness of fit between an operative definition and the concept it intends to measure. In this research the criterion of construct validity has been used. According to some authors, it is the most important because it integrates the considerations of content validity and criterion validity. The criterion of construct validity involves determining the specific mode in which a set of indicators measures one concept and not another. We have used the exploratory factor analysis.

The strategy used to perform the exploratory factor analysis of the proposed scales was solved in three sequential phases, to overcome the conceptual divergence in each of them to continue in the process: 
1- Due to the high number of items in all the scales, we chose to configure a factorial analysis by isolating the items and matching them between the different scales.

2- A Cronbach alpha internal reliability test was performed on each scale that had exceeded the previous phase, and synthetic indexes were constructed summing the scores of the subjects in the items of each scale

3- With the new indicators a new factorial analysis of main components was carried out.

Such an analysis gave rise to the following result. The score of the subjects by scales denote a reduction of dimensions that can be resumed into 5 factors which explain up to $72.61 \%$ of the variance (KMO mean is 0.51 and Bartlett test with p-value $=.000$ ).

The 5 concepts extracted can be named as follows:

1. Reconciliation of familiar tasks. This is the classic concept of performing tasks related to family and child care, linked to people who practice long distance triathlons. In turn it is divided into two scales: hard tasks more specific to the household life, and soft tasks more typical of the public domain.

2. Perception of performance by material. The second factor combines the items referring to the perception of improvement in the performance of the practice of this discipline by the use of the distinctive material elements of the group. This factor is divided into two subscales preferred materials and non-preferred materials.

3. Status generated by achievements. This factor encompasses the subject's level of social acceptance of the environment and satisfaction with achievement.

4. Previous level in the main disciplines. As summarized in the name conjugates the sports that make up the triathlon and the level of the subjects before starting in the realization of this sport.

5. Training and dissemination in social networks. The fifth factor consists of the scales corresponding to amount of training and frequency of publication in social networks.

The second step comprises the reliability analysis of the instrument. In our setting, reliability means the extent to which consistency and replication capacity of the measure (Alaminos Chica, 1998) is reached. To do so, we run the Cronbach Alpha's test (as in Cea D'Ancona, 2001). Reliability tests by gender groups were run and it was elaborated a compact table to test the extent to which factors and subscales have been well-validated. Taking into account that there are no previous research evidence in this field and also nor scales, the interpretation of the data has been done as an exploratory analysis. Thus, the occasion to apply this tool in further research in conditioned to the successful of the present research. 
Construcción y validación de un instrumento para la medición de dimensiones sociales en las prácticas...

Overall, the model outperforms validity and reliability test. However, it presents some weakness in some subscales to analyze gender. Hence, to perform a cross gender analysis in those scales with low reliability it should be necessary to begin with independent items.

\section{INTRODUCCIÓN Y OBJETIVOS}

La población española en los últimos 40 años casi ha doblado el porcentaje en cuanto a práctica deportiva. Históricamente los datos ofrecen una tendencia creciente que se puede diferenciar en dos periodos de unos veinte años. La primera etapa comprende desde el año 75 hasta el 95 donde se da un gran empujón al deporte en nuestro país, la población española pasa de un $22 \%$ a un 39\% de práctica deportiva. El segundo periodo abarca desde el 95 hasta el 2010 donde vemos cierta estabilidad en las cifras, en torno a un 38\%, aunque habría que matizar la evolución de la intensidad y características. Los datos de la última encuesta de hábitos deportivos del 2015 dicen que un 53,5\% de los españoles hacen deporte al menos una vez al año ${ }^{1}$.

Tabla 1. Evolución de porcentaje de la práctica deportiva en España 1975-2010

\begin{tabular}{|l|c|c|c|c|c|c|c|c|}
\hline Año & $\mathbf{1 9 7 5}$ & $\mathbf{1 9 8 0}$ & $\mathbf{1 9 8 5}$ & $\mathbf{1 9 9 0}$ & $\mathbf{1 9 9 5}$ & $\mathbf{2 0 0 0}$ & $\mathbf{2 0 0 5}$ & $\mathbf{2 0 1 0}$ \\
\hline Practica uno o varios deportes & 22 & 25 & 34 & 35 & 39 & 36 & 37 & 40 \\
\hline No practica ninguno & 78 & 75 & 66 & 65 & 61 & 64 & 63 & 60 \\
\hline
\end{tabular}

Fuente: elaboración propia a partir de (García Ferrando, 2006) y estudios de hábitos deportivos del CIS 2198, 2397, 2599 y 2833.

Por otro lado, la evolución y crecimiento del deporte no es proporcional en todos los deportes ni en términos de género. En relación a la desigual evolución de los deportes podemos observar cómo algunas disciplinas individuales consideradas de ultrafondo han tenido un crecimiento muy importante en los últimos 5 años, entre ellas, las carreras de ultratrail y los triatlones de larga distancia.

García Ferrando interpreta el sistema deportivo español haciendo alusión a la aparición de nuevos valores postmaterialistas como el bienestar, la salud, la realización (Durán González, Lagardera Otero, Puig i Barata, \& García Ferrando, 2002), que en parte pueden explicar el crecimiento de estos deportes individuales donde uno de las motivaciones son el reto y la autorrealización por

\footnotetext{
${ }^{1}$ El porcentaje es extraído de la encuesta de hábitos deportivos 2015 que se desarrolló con una metodología diferente al resto de las anteriores coordinadas por García Ferrando. En las anteriores la pregunta hacía referencia a la práctica actual de un deporte, dos o más deportes o ningún deporte, mientras que en la metodología del 2015 la pregunta hace referencia a la práctica deportiva durante el último año, con posibilidad de frecuencia. El dato expuesto del año 2015 corresponde al sumatorio de porcentaje de los individuos que hacen deporte con distinta frecuencia, incluyendo los que hacen deporte aunque sea una vez al año, por ello los resultados son sensiblemente superiores.
} 
el logro de un objetivo. En la siguiente tabla se puede ver la evolución del número de licencias en algunos deportes tradicionales y el crecimiento de los deportes de montaña y triatlón desde el 2011 hasta el 2015 en España².

Tabla 2. Comparativa de licencias federativas entre los años 2011 y 2015.

\begin{tabular}{|l|c|c|c|c|c|c|}
\hline & 2011 & 2012 & 2013 & 2014 & 2015 & $\begin{array}{c}\text { Crecimiento } \\
\text { en } \%\end{array}$ \\
\hline Tenis & 109.418 & 103.898 & 89.830 & 85.668 & 81.581 & $-25,44 \%$ \\
\hline Judo & 108.077 & 106.753 & 106.466 & 104.340 & 104.871 & $-2,97 \%$ \\
\hline Futbol & 834.458 & 869.320 & 855.987 & 874.093 & 909.761 & $9,02 \%$ \\
\hline Baloncesto & 403.561 & 407.728 & 400.153 & 354.949 & 355.845 & $-11,82 \%$ \\
\hline Atletismo & 71.912 & 80.309 & 62.355 & 65.292 & 70.911 & $-1,39 \%$ \\
\hline Ciclismo & 59.717 & 61.733 & 65.339 & 70.800 & 75.855 & $27,02 \%$ \\
\hline Natación & 55.217 & 59.841 & 61.246 & 59.123 & 63.644 & $15,26 \%$ \\
\hline Triatlón & $\mathbf{1 8 . 1 3 7}$ & $\mathbf{2 1 . 0 7 9}$ & $\mathbf{2 4 . 2 4 1}$ & $\mathbf{1 9 . 8 8 4}$ & $\mathbf{2 9 . 7 3 9}$ & $\mathbf{6 3 , 9 7 \%}$ \\
\hline $\begin{array}{l}\text { Escalada y deportes } \\
\text { de montaña }\end{array}$ & $\mathbf{1 4 8 . 5 9 2}$ & $\mathbf{1 5 5 . 9 4 0}$ & $\mathbf{1 6 9 . 1 8 8}$ & $\mathbf{1 8 8 . 2 9 2}$ & $\mathbf{2 0 3 . 8 6 0}$ & $\mathbf{3 7 , 1 9 \%}$ \\
\hline
\end{tabular}

Fuente: elaboración propia a partir de Estadística del deporte federado 2015, Ministerio de educación cultura y deporte.

Por otro lado, la diferencia de práctica deportiva en hombres y mujeres es un hecho evidente que sólo se puede explicar desde una perspectiva amplia multidimensional. Lo cierto es que en el año 2010 el porcentaje de mujeres que practica deportes es un 18\% menor que los hombres, la diferencia en el 2015 es del $13 \%{ }^{3}$. En este aspecto hay dos visiones diferenciadas, una de ellas es la que defiende que las mujeres se incorporan a una cultura deportiva ya definida con rasgos masculinos, y otra argumenta que las mujeres han ido accediendo a esta actividad, y lejos de reproducir los comportamientos masculinos han creado una cultura deportiva propia. (Soler Prat \& Puig i Barata, 2004). Un ejemplo de ello son algunas disciplinas que han tenido una incorporación tardía de la mujer, como es el caso del atletismo y su vinculación negativa con las tareas de reproducción (Iglesias Martínez, Lozano Cabezas, \& Manchado López, 2013). En el ámbito del triatlón, el porcentaje de mujeres que tienen licencia federativa en el 2015 es un 16,8\% sobre los hombres (Estadistica deporte federado, MECD, 2015), aunque en los triatlones de larga distancia el porcentaje de participación apenas representa el $10 \%$.

\footnotetext{
${ }^{2}$ Los datos expuestos hacen referencia al número de licencias en Deportes de montaña y escalada y en Triatlón. En el caso de carreras de montaña (ultratrails) estarían reflejadas dentro de deportes de montaña y escalada. El triatlón de larga distancia está reflejado en las licencias de triatlón.

${ }^{3}$ La diferencia entre los datos del 2010 y el 2015 sólo son interpretables si se tienen en cuenta la diferencia de metodología empleada, como ya se ha señalado anteriormente.
} 
Desde la perspectiva funcionalista algunos autores consideran el deporte como una manifestación o subsistema del sistema social imperante (Durán González et al., 2002). Por otra parte Althusser lo presenta como un aparato ideológico de Estado, un conjunto de múltiples instituciones diferenciadas y especializadas que, al concentrar y transmitir la ideología dominante, reproduce las existentes relaciones de dominación (Barberó Gonzalez, 1991) . Teniendo en cuenta estas premisas se puede intuir que las diferencias de género en el deporte manifiestan las desigualdades latentes en otros ámbitos sociales.

En este contexto y aludiendo al concepto de "hábitus" de Bourdieu, para explicar la posición social de un grupo y sus prácticas, (Bourdieu, 1987) y "sentimiento de comunidad compartido" de Maffesoli (Carretero Pasín, 2003) se plantea la investigación en curso sobre la que versa este texto, que focaliza la atención hacia un intento por diseñar instrumentos de medición capaces de captar estas dimensiones sociales de la práctica deportiva.

El principal objetivo es la descripción social del estilo de vida de una subcultura en el ámbito deportivo, concretamente los deportistas de ultrafondo, y dentro de estos, los triatletas de larga distancia, introduciendo en este análisis una perspectiva de género de manera transversal con el fin de apreciar las diferencias de opinión y comportamiento entre hombres y mujeres.

\section{METODOLOGÍA: DATOS Y CUESTIONARIO}

La fuente empírica de la investigación se centra en la realización de una encuesta. Dicha encuesta fue aplicada al subgrupo social de cultura deportiva compuesto por deportistas de larga distancia que practican triatlón y participan activamente en esta disciplina. Para ello se seleccionaron dos pruebas del calendario nacional que forman parte de la copa nacional en la especialidad de larga distancia, donde una vez eliminadas duplicidades el marco muestral quedó fijado en 1800 sujetos. A partir de él se procedió a un muestreo no probabilístico derivado de la modalidad de aplicación del cuestionario (autoadministrado por Internet), quedando una muestra final de 270 casos, de los que tras la depuración resultaron 230 que respondieron a todas las variables propuestas, requisito imprescindible para la construcción de escalas dentro del cuestionario.

Por su parte el diseño del cuestionario siguió una lógica de integración metodológica, con una primera fase exploratoria de carácter cualitativo a través de entrevistas en profundidad que permitieron establecer categorías de algunas variables subjetivas, y determinar cuáles eran los indicadores más adecuados para dimensiones y/o conceptos más generales. Se realizaron 6 entrevistas en profundidad a cuatro hombres y dos mujeres elegidos de forma intencional por el criterio de implicación en el fenómeno a estudiar. Las personas entrevistadas, además de participar y/o haber participado con anterioridad en pruebas de larga distancia ocupan un lugar de informantes estratégicos dentro de la 
población objeto de estudio que les permite una capacidad de observación y analítica continuada: promotores y organizadores de pruebas, gerencia de marcas, presidencia/dirección de clubs (club femenino), y participación como jueces de triatlón de larga distancia.

Una vez diseñado el cuestionario, se procedió a la aplicación de un pretest del instrumento a un subgrupo de casos que cumplían el perfil específico de la muestra, lo cual completó el proceso de construcción, quedando éste con una estructura final que integraba un total de 69 preguntas. Los bloques temáticos y contenidos del cuestionario de forma resumida en la tabla no 3 .

\section{ANÁLISIS DE LA VALIDEZ Y FIABILIDAD DEL INSTRUMENTO}

Del total de preguntas planteadas, se ha sometido a criterios de validez y fiabilidad las escalas compuestas por distintos ítems agrupados, siguiendo para ello estrategias empíricas de contrastación sobre los resultados obtenidos tras la producción y recogida de la información. Entendemos por validez la congruencia o bondad de ajuste entre una definición operativa y el concepto que pretende medir (Alaminos, 1998; Alaminos, Francés, Penalva y Santacreu, 2015), la relación entre el concepto teórico y el indicador empírico que finalmente aplicaremos en la realidad. En la investigación se debe comprobar si los indicadores elegidos realmente miden el significado que hemos dado en el concepto teórico (Cea D'Ancona, 2001). En la encuesta la validez del instrumento está relacionada con la calidad del proceso de operacionalización. Por tanto en los análisis de validez pretendemos evaluar el trabajo de operacionalización con el fin de minimizar los errores. Para este trabajo se ha utilizado el criterio de "validez de constructo", que implica determinar el modo específico en que un conjunto de indicadores miden un concepto determinado y no otro. Aquí el grado de validez se determina, como se ha señalado anteriormente, a partir de métodos empíricos, no teóricos. Quizás el método más utilizado para la validez de constructo es el del análisis factorial exploratorio, que es utilizado sobre todo en el caso de escalas multidimensionales. Para algunos autores la validez de constructo es la más importante dentro de los distintos tipos ya que integra las consideraciones de validez de contenido y de criterio en un marco común para probar hipótesis sobre las relaciones teóricamente relevantes (Chacón Moscoso, Pérez Gil, \& Moreno Rodríguez, 2000). Con este procedimiento exploramos el número de factores subyacente a una escala de indicadores sin una hipótesis previa. ${ }^{4}$ De esta forma es posible determinar el número de dimensiones que generan la

\footnotetext{
${ }^{4}$ Debemos matizar que en el acercamiento al hecho social encontramos la dificultad de analizar los factores con carácter puramente exploratorio ya que se entremezcla el carácter confirmatorio en algunos casos, fruto de las conexiones que se establecen previamente sobre el objeto de estudio.
} 
Construcción y validación de un instrumento para la medición de dimensiones sociales en las prácticas...

escala y comprobar si tanto este número como su estructura coinciden con el proceso de operacionalización llevado a cabo para la generación de ítems.

Tabla 3. Estructura y principales contenidos registrados en el cuestionario

\begin{tabular}{|l|l|}
\hline Bloques & Principales variables \\
\hline Perfil deportivo & $\begin{array}{l}\text { Trayectoria en principales disciplinas } \\
\text { Motivaciones para inicio y mantenimiento en el grupo }\end{array}$ \\
\hline $\begin{array}{l}\text { Conciliación practica } \\
\text { triatlón de larga distancia } \\
\text { con tareas en el hogar }\end{array}$ & $\begin{array}{l}\text { Diferenciación por tareas y quien es la persona que las } \\
\text { realiza } \\
\text { Escala percepción de satisfacción de la pareja }\end{array}$ \\
\hline $\begin{array}{l}\text { Conciliación práctica } \\
\text { triatlón de larga distancia } \\
\text { con el cuidado de hijos } \\
\text { menores de 12 años }\end{array}$ & $\begin{array}{l}\text { Diferenciación de actividades realizadas y quien es la } \\
\text { persona que las realiza } \\
\text { Escala percepción de satisfacción de la pareja }\end{array}$ \\
\hline $\begin{array}{l}\text { Deporte en el entorno } \\
\text { Preparación en larga } \\
\text { distancia }\end{array}$ & $\begin{array}{l}\text { Deporte practicado entorno cercano, como forma de } \\
\text { transferencia } \\
\text { Escala aceptación del entorno } \\
\text { Práctica deportiva en general }\end{array}$ \\
$\begin{array}{l}\text { Horas disponibles al día } \\
\text { Dedicación entrenamiento por disciplina } \\
\text { Mejores resultados y nivel de satisfacción } \\
\text { Inversión económica entrenamiento }\end{array}$ \\
\hline $\begin{array}{l}\text { Participación y viajes en } \\
\text { larga distancia }\end{array}$ & $\begin{array}{l}\text { Escala percepción/inclusión social viajes } \\
\text { Grupos con los que se comparte } \\
\text { Coste viajes }\end{array}$ \\
\hline $\begin{array}{l}\text { Perfil del grupo específico } \\
\text { de larga distancia }\end{array}$ & $\begin{array}{l}\text { Elementos diferenciadores } \\
\text { Percepción de rendimiento por uso material distintivo } \\
\text { Precio dos elementos distintivos: neopreno/ bici competición } \\
\text { Escala de publicación en redes sociales } \\
\text { Fuentes de información }\end{array}$ \\
\hline $\begin{array}{l}\text { Sexo, edad, estado civil, nivel de estudios alcanzado, } \\
\text { número de hijos, jornada laboral, ocupación, ingresos netos } \\
\text { y familiares, comunidad autónoma y situación de } \\
\text { convivencia }\end{array}$ \\
\hline
\end{tabular}

Las escalas propuestas para validar fueron las siguientes:

A. Escala de conciliación tareas del hogar "duras" predominancia ámbito privado.

B. Escala de conciliación tareas del hogar "blandas" predominancia ámbito público.

C. Escala de nivel previo de disciplinas antes de practicar triatlón de larga distancia.

D. Escala de aceptación social.

E. Nivel (cantidad) de entrenamiento ponderado. 
F. Nivel de satisfacción de resultados.

G. Escala de uso de material identificativo.

H. Percepción de rendimiento por material "preferente" (indispensable) de triatlón de larga distancia.

I. Percepción de rendimiento por material "no preferente" (no indispensable) de triatlón de larga distancia.

J. Escala de gasto en conceptos derivados de la práctica.

K. Frecuencia de publicaciones en RR.SS sobre triatlón de larga distancia.

La estrategia específica implementada para realizar el análisis factorial exploratorio de las escalas propuestas se resolvió en tres fases secuenciales, de tal manera que los análisis debían superar la divergencia conceptual en cada una de ellas para seguir en el proceso. Las fases son las siguientes:

1- Dado el elevado número de ítems que integran la totalidad de las escalas (aproximadamente 40) se ha optado por configurar la prueba factorial inicial aislando los ítems de cada escala y contrastando su dimensionalidad de forma pareada con el resto de ítems de las distintas escalas. En esta primera fase se desestimaron dos escalas, correspondientes a "uso de materiales identificativos" en el ámbito objeto de estudio y "gasto en conceptos derivados de la práctica" (escalas G y J enunciadas anteriormente).

2- Realización del test de fiabilidad interna alfa de Cronbach en cada escala $\mathrm{y}$, en el caso de que el resultado fuera satisfactorio en términos estadísticos, construcción de índices sintéticos para cada escala. Dada la naturaleza ordinal (no cuantitativa) de la mayor parte de los ítems utilizados, para la elaboración de estos índices sintéticos se ha recurrido al sumatorio de la puntuación de los sujetos en los ítems de cada escala.

3- Análisis factorial exploratorio a partir de componentes principales de las nuevas variables creadas en la segunda fase.

El resultado resumen del proceso de validez se observa en la tabla 4 (Varianza explicada en el análisis factorial):

${ }^{5}$ Para el análisis factorial se utilizó el programa estadístico IBM-SPSS 22 
Tabla 4. Varianza explicada en el análisis factorial

\begin{tabular}{|c|c|c|c|c|}
\hline \multirow[b]{2}{*}{ Componente } & $\begin{array}{c}\text { Sumas de } \\
\text { extracción de } \\
\text { cargas al } \\
\text { cuadrado }\end{array}$ & \multicolumn{3}{|c|}{ Sumas de rotación de cargas al cuadrado } \\
\hline & $\%$ acumulado & Total & $\%$ de varianza & $\begin{array}{c}\% \\
\text { acumulado }\end{array}$ \\
\hline 1 & 18,443 & 1,546 & 17,174 & 17,174 \\
\hline 2 & 36,282 & 1,411 & 15,678 & 32,852 \\
\hline 3 & 50,396 & 1,369 & 15,213 & 48,066 \\
\hline 4 & 62,959 & 1,157 & 12,851 & 60,917 \\
\hline 5 & 72,613 & 1,053 & 11,696 & 72,613 \\
\hline 6 & & & & \\
\hline 7 & & & & \\
\hline 8 & & & & \\
\hline 9 & & & & \\
\hline
\end{tabular}

Tabla 5. Matriz de puntuaciones en componentes rotados

\begin{tabular}{|c|c|c|c|c|c|}
\hline & \multicolumn{5}{|c|}{ Componente } \\
\hline & 1 & 2 & 3 & 4 & 5 \\
\hline ConciliaTareasDuras & 805 &, 130 &,- 135 &, 060 &,- 049 \\
\hline ConciliaTareasBlandas & 844 &, 025 & ,072 &,- 010 &,- 077 \\
\hline PerRendMateriaNOindisp & ,094 & ,718 &,- 258 &,- 043 &, 360 \\
\hline PerRendMaterialIndisp & ,132 &, 805 & ,203 &,- 009 &,- 097 \\
\hline AceptacionSocial &, 110 &,- 006 & ,688 & ,458 & , 165 \\
\hline SatisfaccionResultados &,- 119 & ,068 & ,816 &,- 190 &,- 072 \\
\hline NivelPrevioNatBiciCarrera & ,018 &,- 011 &,- 032 & ,935 &,- 009 \\
\hline NivelEntrenoPonderado &,- 276 & ,452 & ,291 & ,161 &,- 531 \\
\hline FrecPublRRSS &,- 238 & ,144 & ,115 &, 074 & ,769 \\
\hline
\end{tabular}

Método de extracción: análisis de componentes principales. Análisis factorial con rotación varimax, adecuación y esfericidad $\mathrm{KMO}=0,51$, y prueba de esfericidad de Bartlett con Sig.= ,000).

La puntuación de los individuos en las escalas evidencia una reducción de dimensiones que se pueden resumir en 5 factores que explican un $72 \%$ de la varianza haciendo referencia a 5 conceptos que los podemos denominar de la siguiente manera:

1. Conciliación tareas familiares. Se trata del concepto clásico de la realización de las tareas vinculadas al ámbito familiar y cuidado de los hijos, ligado a las personas que practican triatlón de larga distancia. El enfoque que 
presenta este concepto está contextualizado en "periodos en los que se está entrenando/preparando pruebas de triatlón de larga distancia".

2. Percepción del rendimiento por material. El segundo factor aúna los ítems referentes a la percepción de mejorar en el rendimiento de la práctica de esta disciplina por el hecho de usar los elementos materiales distintivos del grupo.

3. Estatus generado por logros. Este factor engloba el nivel de aceptación social del entorno del sujeto con la satisfacción por los logros conseguidos. Por una parte explica la consecución de logro/reto individual que conllevan las características de los deportes de ultrafondo ligado al giro hacia los valores postmaterialistas. Por otro lado explica la aceptación social o estatus conseguido por el hecho de practicar este deporte.

4. Nivel previo en las principales disciplinas. Como se resume en el nombre conjuga los deportes que componen el triatlón y el nivel de los sujetos antes de iniciarse en la realización de este deporte.

5. Entrenamiento y difusión en redes sociales. El quinto factor se compone de la cantidad de entrenamiento y la frecuencia de publicación en las redes sociales.

A su vez cada uno de estos factores contiene subescalas identificativas del mismo:

1. Primer factor: conciliación tareas familiares, que a su vez podemos ver como se valida en dos escalas distintas denominadas:

a. Escala de conciliación tareas del hogar "duras" predominancia ámbito privado. Se han obtenido agrupaciones de los ítems que componen este concepto que son los referentes a la realización de tareas tradicionales de limpieza, cocina, ropa, actividades con los hijos dentro del hogar, cuidados médicos de los hijos y actividades extraescolares.

b. Escala de conciliación tareas del hogar "blandas" predominancia ámbito público. Las tareas que contienen puntuaciones conjuntas son las referentes a la compra, las gestiones y papeleos, el bricolaje y la dedicación a los hijos fuera del hogar.

2. Segundo factor: percepción del rendimiento por material identificativo del grupo de pertenencia, que a su vez se valida en dos escalas distintas:

a. Percepción de rendimiento por material "preferente" (indispensable) de triatlón de larga distancia. Hemos encontrado relación en las puntuaciones que se ofrecen sobre materiales que se consideran más importantes para el entrenamiento y planificación de la práctica de la disciplina deportiva, estos son el plan de entrenamiento, fisioterapia, suplementación alimenticia, pulsómetro/gps y la visera. 
b. Percepción de rendimiento por material "no preferente" (no indispensable) de triatlón de larga distancia. El otro conjunto de indicadores que nos muestra el análisis es el referente a aquellos elementos relacionados con el estereotipo o imagen material que pueden influir en el rendimiento sin ser necesarios $o$ indispensables, estos son el casco aero, las prendas de compresión, la cabra, las ruedas de perfil y el potenciómetro.

3. Tercer factor: estatus generado por los logros, que integra los conceptos:

a. Escala de aceptación social. La escala de aceptación social agrupa las puntuaciones que reciben los sujetos en aceptación social por parte del entorno cercano, concretamente hace referencia a opinión de los padres, los hermanos, la pareja, los compañeros de trabajo y los amigos.

b. Nivel de satisfacción de resultados. El nivel de satisfacción de resultados combina la satisfacción de los triatletas sobre las marcas conseguidas en ironman, en medio ironman y el nivel de satisfacción general de los resultados en la práctica de este deporte.

4. Cuarto factor: nivel previo en los deportes que componen el triatlón:

a. Escala de nivel previo de disciplinas antes de practicar triatlón de larga distancia. En este concepto se mide el nivel obtenido con anterioridad a la práctica de triatlón de larga distancia, en las disciplinas que componen el triatlón, que son la natación, el ciclismo y carrera pie, dentro de atletismo.

5. Quinto factor: el entrenamiento y su difusión en redes sociales, que está compuesto por dos escalas:

a. Frecuencia de publicaciones en RR.SS sobre triatlón de larga distancia. A través de esta subescala se pretende medir el la frecuencia de publicaciones que tengan relación con la disciplina deportiva triatlón de larga distancia en las tres principales redes sociales.

b. Nivel (cantidad) de entrenamiento ponderado. La escala de nivel de entrenamiento ponderado combina el sumatorio de las distancias que entrenan los individuos en kilómetros realizados a la semana en las disciplinas que componen el triatlón, con un grado de ponderación, es decir, estableciendo equivalencias entre los $\mathrm{km}$ de ciclismo y de natación sobre carrera a pie como valor de referencia.

Los ítems que componen cada uno de los factores y las escalas se muestran en el anexo 1.

Una vez realizado el proceso de descomposición de factores y validación en término de dimensiones se ha procedido al análisis de fiabilidad interna en las escalas resultantes. El concepto de fiabilidad expresa la consistencia y reproductibilidad de una medición (Alaminos Chica, 1998). Como método para 
comprobar la fiabilidad a partir de estabilidad y consistencia interna de las escalas se ha llevado a cabo pruebas de alfa de Cronbach, uno de los más utilizados por los investigadores en los últimos años (Cea D'Ancona, 2001). Dichas pruebas del análisis se han aplicado tanto sobre el total de la muestra de manera agregada como por subgrupos hombres y mujeres, considerando importante la transversalidad de género que se plantean en los objetivos. El resultado de este análisis se recoge en la tabla $6 .^{6}$

Tabla 6. Puntuaciones de alfa de Cronbach en factores por grupos hombres y

\begin{tabular}{|c|c|c|c|}
\hline \multicolumn{4}{|c|}{ mujeres } \\
\hline \multirow{2}{*}{ FACTORES } & \multicolumn{3}{|c|}{ Puntuación Alfa de Cronbach } \\
\hline & Total & Mujeres & Hombres \\
\hline A. Conciliación tareas familiares &, 519 & ,712 & ,406 \\
\hline $\begin{array}{l}\text { Escala de conciliación } \\
\text { entrenamiento de larga } \\
\text { distancia con "Tareas duras" }\end{array}$ & ,676 & 861 &, 597 \\
\hline $\begin{array}{l}\text { Escala de conciliación } \\
\text { entrenamiento de larga } \\
\text { distancia con "Tareas } \\
\text { blandas" }\end{array}$ &, 501 & 425 &, 537 \\
\hline $\begin{array}{l}\text { B. Percepción del rendimiento por } \\
\text { material identificativo }\end{array}$ & ,780 &, 507 & .798 \\
\hline $\begin{array}{l}\text { Escala percepción de } \\
\text { incremento de nivel a través } \\
\text { del material "indispensable" }\end{array}$ & ,701 &, 582 & ,723 \\
\hline $\begin{array}{l}\text { Escala percepción de } \\
\text { incremento de nivel a través } \\
\text { del material "NO } \\
\text { indispensable" }\end{array}$ & ,735 &, 555 & ,748 \\
\hline C. Estatus generado por los logros & ,572 & 469 & ,606 \\
\hline $\begin{array}{l}\text { Escala de satisfacción } \\
\text { resultados. }\end{array}$ & ,462 & ,635 & 434 \\
\hline $\begin{array}{l}\text { Escala aceptación entorno } \\
\text { social }\end{array}$ & 654 & ,604 & ,660 \\
\hline $\begin{array}{l}\text { D. Nivel previo en los deportes que } \\
\text { componen el triatlón }\end{array}$ &, 551 &, 551 &, 548 \\
\hline $\begin{array}{l}\text { E. Entrenamiento y su difusión en } \\
\text { redes sociales }\end{array}$ &, 555 & ,387 &, 587 \\
\hline $\begin{array}{l}\text { Escala de entrenamiento } \\
\text { ponderado. }\end{array}$ & ,632 & 482 & 657 \\
\hline $\begin{array}{l}\text { Escala publicación en redes } \\
\text { sociales }\end{array}$ & ,743 & ,744 & ,742 \\
\hline
\end{tabular}

${ }^{6}$ Para el análisis de fiabilidad interna se utilizó el programa estadístico IBM-SPSS 22 


\section{CONCLUSIONES Y DISCUSIÓN}

El objetivo principal de este trabajo era validar una serie de escalas para el uso en el análisis de características sociales en una subcultura específica de creciente interés dentro del escenario deportivo actual, el triatlón de larga distancia. El proceso de integración metodológica en la búsqueda de sinergias para la construcción de conceptos y redacción de las variables empíricas cerradas o semicerradas, así como el requerimiento técnico del pretest utilizado en el cuestionario han dado lugar a los indicadores ya expuestos . A través de la técnica de análisis factorial exploratorio en la búsqueda de validez y alfa de Cronbach por su parte en cuanto a fiabilidad interna de respuesta se han validado una serie de factores, que a su vez aglutinan subescalas más específicas de tal manera que en el análisis posterior se podrán utilizar los conceptos globales o las dimensiones de cada uno de ellos. La posibilidad de utilizar este instrumento para en futuras ocasiones también está condicionado por el proceso aquí descrito. Como resumen se ha elaborado la siguiente tabla que contiene los indicadores de validez y de fiabilidad:

Tabla 7. Resumen combinado de los resultados en las pruebas de fiabilidad y validez

\begin{tabular}{|c|c|c|c|c|c|c|c|c|c|}
\hline & & \multicolumn{3}{|c|}{ Fiabilidad } & \multicolumn{5}{|c|}{ Validez } \\
\hline & $\begin{array}{l}\text { oo de } \\
\text { Items } \\
\end{array}$ & Alfa & $\begin{array}{c}\text { Alfa } \\
\text { M }\end{array}$ & \begin{tabular}{|c|} 
Alfa \\
$\mathrm{H}$ \\
\end{tabular} & F1 & $\mathrm{F} 2$ & F3 & $\mathrm{F} 4$ & F5 \\
\hline A. Conciliación tareas familiares & 10 & ,519 & ,712 & ,406 & & & & & \\
\hline $\begin{array}{l}\text { Escala de conciliación entrenamiento } \\
\text { de larga distancia con "Tareas duras" }\end{array}$ & 6 & ,676 & 861 & ,597 & ,805 & & & & \\
\hline $\begin{array}{l}\text { Escala de conciliación entrenamiento } \\
\text { de larga distancia con "Tareas blandas" }\end{array}$ & 4 & ,501 & ,425 & ,537 & 844 & & & & \\
\hline $\begin{array}{l}\text { B. Percepción del rendimiento por material } \\
\text { identificativo }\end{array}$ & 10 & ,780 & ,507 & .798 & & & & & \\
\hline $\begin{array}{l}\text { Escala percepción de incremento de } \\
\text { nivel a través del material } \\
\text { "indispensable" }\end{array}$ & 5 & 701 & ,582 & ,723 & & ,718 & & & \\
\hline $\begin{array}{l}\text { Escala percepción de incremento de } \\
\text { nivel a través del material " NO } \\
\text { indispensable" }\end{array}$ & 5 & ,735 & ,555 & ,748 & & ,805 & & & \\
\hline C. Estatus generado por los logros & 8 & ,572 & 469 & ,606 & & & & & \\
\hline Escala de satisfacción resultados. & 3 & ,462 & 635 & ,434 & & & 688 & & \\
\hline Escala aceptación entorno social & 5 & ,654 & 604 & ,660 & & & ,816 & & \\
\hline $\begin{array}{l}\text { D. Nivel previo en los deportes que componen } \\
\text { el triatlón }\end{array}$ & 3 &, 551 & ,551 & ,548 & & & & ,935 & \\
\hline $\begin{array}{ll}\text { E. Entrenamiento y su difusión en redes } \\
\text { sociales }\end{array}$ & 6 & ,555 & ,387 &, 587 & & & & & \\
\hline Escala de entrenamiento ponderado. & 3 & ,632 &, 482 & ,657 & & & & & -531 \\
\hline Escala publicación en redes sociales & 3 & ,743 & 744 & ,742 & & & & & ,769 \\
\hline
\end{tabular}

Método de extracción: análisis de componentes principales. Análisis factorial con rotación varimax, adecuación y esfericidad $\mathrm{KMO}=$ 0,51, y prueba de esfericidad de Bartlett Chi-cuadrado 121,627 (gl 36 y Sig. ,000). 
La propia estructura de la tabla corresponde al proceso de construcción/extracción y corrección en los factores que hacen referencia a conceptos multidimensionales ya validados a partir del análisis factorial. La representación gráfica de forma combinada con los test posteriores de alfa de Cronbach nos ofrece un resumen entre validez y fiabilidad que nos permite ver los puntos débiles del modelo, por lo que debemos pasar a comentar las puntuaciones de fiabilidad, que nos dirán qué escalas y/o subescalas se validan de forma completa, y cuales se validan en el grupo de hombres y de mujeres.

Teniendo en cuenta que las escalas construidas tienen pocos ítems, como sugiere Loewenthal, un valor de fiabilidad de 0.6 puede ser considerado aceptable para escalas con menos de 10 ítems. De la misma forma Nunnally concluye que en las primeras fases de la investigación o estudios exploratorios un valor de fiabilidad de 0,6 o 0,5 puede ser suficiente.

Debemos considerar que no existen estudios previos en este campo, y de forma específica no se han elaborado escalas por lo que la interpretación de los datos se ha realizado con carácter exploratorio y no confirmatorio en una fase inicial de acercamiento al objeto de estudio considerando fiables las escalas a partir de 0,5. Siguiendo estas pautas las conclusiones son las siguientes:

A. La escala de "Conciliación de tareas familiares" está validada y puede ofrecer un análisis interesante, ya que esta disciplina deportiva requiere una inversión de tiempo muy alta, aunque no podemos utilizar la puntuación en caso de las mujeres porque no tenemos muestra de fiabilidad. Dentro de ella, la subescala "conciliación tareas duras" que está compuesta por los ítems determinantes de las tradicionales tareas del ámbito privado sí que se pueden considerar válidas las puntuaciones y se podrán establecer diferencias entre grupos de hombres y mujeres.

B. La segunda escala aglutina la percepción subjetiva del incremento del rendimiento a través de elementos que se han ido configurando como identificativos del grupo objeto de estudio, es decir, que aunque son materiales/servicios comunes a diferentes deportes se usan en mayor medida en este contexto deportivo y tienen un componente simbólico importante, incrementen o no el rendimiento. Se ha validado tanto el concepto general como las dos subescalas que lo componen. Las dos subescalas disgregan el total de elementos en la consideración de "indispensables" o preferentes, que son aquellos materiales/servicios que pueden aportar más beneficios o se configuran como más necesarios por las características específicas de la disciplina. Por otro lado será interesante el análisis de la subescala que hace referencia a la "percepción del incremento de nivel por material no indispensable", no preferente, que es aquel más superfluo, que representa más la imagen del estereotipo y menos el rendimiento. 
C. En términos generales se valida la escala de estatus generado por logros, aunque con limitaciones de fiabilidad en caso de las puntuaciones de las mujeres. Este concepto hace referencia a la conjunción del grado de aceptación social del entorno cercano del individuo (externo al propio grupo deportivo) con la satisfacción por los resultados obtenidos en las marcas/tiempos en pruebas de larga distancia. En cuanto a las subescalas que componen este concepto de forma independiente la escala de satisfacción de resultados no presenta indicadores de fiabilidad. Por parte de la escala de aceptación social queda validada y se podrá utilizar en análisis independientes y comparativos entre hombres y mujeres.

D. En relación a la escala de nivel previo en los deportes que componen el triatlón, dado que nos hallamos ante un concepto bastante sencillo, ésta ha tratado de agrupar la práctica previa y nivel de los individuos en las tres disciplinas que componen el triatlón, natación, ciclismo y carrera a pie. Las puntuaciones en el estadístico alfa de Cronbach indican que se podrá usar en los análisis del conjunto de la muestra y en los subgrupos hombres y mujeres.

E. Por último podemos decir que la escala entrenamiento y difusión en RR.SS. llama la atención en los resultados obtenidos. Las pruebas de fiabilidad arrojan puntuaciones que son aceptables en el conjunto pero no en el caso de las mujeres. Encontramos una significación negativa en el nivel de entrenamiento ponderado y positiva en el nivel de publicaciones en RR.SS. Aunque no implica causalidad refleja puntuaciones bajas en entrenamiento ponderado junto a puntuaciones altas en publicación en RR.SS. Por su parte, la escala de nivel de entrenamiento ponderado no es fiable para el análisis del grupo de mujeres.

Para el análisis transversal de la perspectiva de género en las escalas que presentan niveles bajos de fiabilidad se deberá realizar a partir de los ítems de manera independiente.

Cabe destacar que la mejora en el testado de validez y fiabilidad de las escalas propuestas estaría sujeto a futuros análisis test-pretest, además de utilizar muestras más grandes con una proporción de mujeres más alta. 
Eloy García García

\section{BIBLIOGRAFÍA}

Alaminos Chica, A. (1998). Teoría y práctica de la encuesta. Aplicación en los países en vias de desarrollo (Editorial). Alicante.

Alaminos Chica, A., Francés García, F. J., Penalva Verdú, C., \& Santacreu Fernández, Ó. A. (2015). Análisis multivarianate para las ciencias sociales I (Pydlos). Cuenca.

Barberó Gonzalez, J. I. (1991). Sociología del deporte, configuración de un campo. Revista de Educación, 295.

Bourdieu, P. (1987). Cosas dichas (Gedisa). Barcelona.

Carretero Pasín, Á. E. (2003). La noción de imaginario social en Michel Maffesoli. Revista Española de Investigaciones Sociológicas, (104), 199-209.

Cea D’Ancona, M. ángeles. (2001). Metodología cuantitativa. Estrategias y técnicas de investigación social (Sintesis). Madrid.

Chacón Moscoso, S., Pérez Gil, J. A., \& Moreno Rodríguez, R. (2000). Validez de constructo: el uso del análisis factorial exploratorio-confirmatorio para obtener evidencias de validez. Psicothema, ISSN 0214-9915, Vol. 12, No. Extra 2, 2000, 12(2), 442-446.

Díaz de Rada, V. (2009). Analisis de datos de encuesta. Desarrollo de una investigación completa utilizando SPSS (Gedisa). Barcelona.

Díaz de Rada, V. (2012). Ventajas e inconvenientes de la encuesta por Internet. Papers, 97/1, 193-223.

Durán González, J., Lagardera Otero, F., Puig i Barata, N., \& García Ferrando, M. (2002). Sociología del deporte (Alianza Ed). Madrid.

García Ferrando, M. (2006). Veinticinco años de análisis del comportamiento deportivo de la población española (1980-2005). Revista Internacional de Sociología, 64(44), 15-38. http://doi.org/10.3989/ris.2006.i44.26

Iglesias Martínez, M. J., Lozano Cabezas, I., \& Manchado López, M. del C. (2013). Deporte e igualdad: las voces de las deportistas de élite. Feminismo/s, (21). http://doi.org/10.14198/fem.2013.21.05

MECD. (2015). Estadística de Deporte Federado 2015. Ministerio de Educación Cultura y Deporte.

Soler Prat, S., \& Puig i Barata, N. (2004). Mujer y deporte en España: estado de la cuestión y propuesta interpretativa. Apunts: Educación Física Y Deportes, ISSN 1577-4015, No 76, 2004, (76), 71-78. 
Construcción y validación de un instrumento para la medición de dimensiones sociales en las prácticas...

\section{ANEXO 1. Relación de variables empíricas que componen los factores y las subescalas.}

FACTOR: Nivel previo en los deportes que componen el triatlón (compuesto por una subescala)

¡¿Cuál era tu nivel en los siguientes deportes antes de empezar a hacer triatlón?

\begin{tabular}{cccccc}
\hline $\begin{array}{c}\text { No lo había } \\
\text { practicado } \\
\text { nunca }\end{array}$ & $\begin{array}{c}\text { Lo había } \\
\text { practicado } \\
\text { una vez }\end{array}$ & $\begin{array}{c}\text { Lo había } \\
\text { practicado } \\
\text { como } \\
\text { aficionado }\end{array}$ & $\begin{array}{c}\text { Lo había } \\
\text { practicado } \\
\text { como } \\
\text { federado }\end{array}$ & $\begin{array}{c}\text { Lo había } \\
\text { practicado } \\
\text { como } \\
\text { profesional }\end{array}$ & No contesta \\
\hline 1 & 2 & 3 & 4 & 5 & 9 \\
\hline 1 & 2 & 3 & 4 & 5 & 9 \\
\hline
\end{tabular}

FACTOR: Percepción del rendimiento por material identificativo.

Subescala percepción de rendimiento por material "preferente"

-De la siguiente lista de elementos, indica en tu opinión CUÁNTO influyen en el rendimiento: 1 significa que no influye NADA en el rendimiento y 5 que influye MUCHO en el rendimiento.

\begin{tabular}{lccccccc}
\cline { 2 - 7 } & Nada & Poco & $\begin{array}{c}\text { Término } \\
\text { medio }\end{array}$ & Bastante & Mucho & NS & NC \\
\hline Plan de entrenamiento & 1 & 2 & 3 & 4 & 5 & 8 & 9 \\
\hline Pulsómetro/GPS & 1 & 2 & 3 & 4 & 5 & 8 & 9 \\
\hline Visera & 1 & 2 & 3 & 4 & 5 & 8 & 9 \\
\hline $\begin{array}{l}\text { Suplementación } \\
\text { alimenticia }\end{array}$ & 1 & 2 & 3 & 4 & 5 & 8 & 9 \\
\hline Fisioterapia/masajes & 1 & 2 & 3 & 4 & 5 & 8 & 9 \\
\hline
\end{tabular}

-De la siguiente lista de elementos, indica en tu opinión CUÁNTO influyen en el rendimiento: 1 significa que no influye NADA en el rendimiento y 5 que influye MUCHO en el rendimiento.

\begin{tabular}{lccccccc}
\cline { 2 - 8 } & Nada & Poco & $\begin{array}{c}\text { Término } \\
\text { medio }\end{array}$ & Bastante & Mucho & NS & NC \\
\hline Casco aero & 1 & 2 & 3 & 4 & 5 & 8 & 9 \\
\hline Cabra & 1 & 2 & 3 & 4 & 5 & 8 & 9 \\
\hline Ruedas de perfil & 1 & 2 & 3 & 4 & 5 & 8 & 9 \\
\hline Prendas de compresión & 1 & 2 & 3 & 4 & 5 & 8 & 9 \\
\hline Potenciómetro & 1 & 2 & 3 & 4 & 5 & 8 & 9 \\
\hline
\end{tabular}


FACTOR: Entrenamiento y su difusión en redes sociales.

Subescala frecuencia de publicaciones en redes sociales

- ¿Con qué frecuencia utilizas las siguientes redes sociales para publicaciones relacionadas con tu vida deportiva? (Entrenamientos, competiciones, objetivos...etc)

\begin{tabular}{lccccccc} 
& Diariamente & $\begin{array}{c}\text { Cada 2- } \\
\text { 3 días }\end{array}$ & Semanalmente & $\begin{array}{c}\text { Cada dos } \\
\text { semanas }\end{array}$ & Mensualmente & No uso & NC \\
\hline Facebook & 1 & 2 & 3 & 4 & 5 & 6 & 9 \\
\hline Twitter & 1 & 2 & 3 & 4 & 5 & 6 & 9 \\
\hline Instagram & 1 & 2 & 3 & 4 & 5 & 6 & 9 \\
\hline
\end{tabular}

\section{Subescala entrenamiento ponderado}

En una semana de intensidad media, ¿cómo distribuyes tu tiempo de entrenamiento?

-Km realizados de NATACIÓN en una semana (de media)

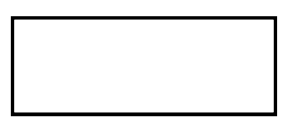

-Km realizados de CICLISMO en una semana (de media)

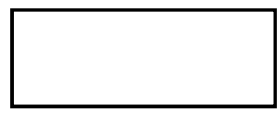

-Km realizados de CARRERA A PIE en una semana (de media)

\section{FACTOR: Conciliación tareas familiares:}

Subescala conciliación entrenamiento larga distancia con tareas duras

-En una semana normal de entrenamiento de larga distancia, EN TU HOGAR/ EN CUANTO A LA ATENCIÓN DE LOS HIJOS ¿quién realiza las siguientes tareas principalmente? (mover la barra hacia la derecha para ver todas las opciones)

\begin{tabular}{|c|c|c|c|c|c|c|}
\hline & Yo & Mi pareja & $\begin{array}{c}\text { De forma } \\
\text { compartida }\end{array}$ & $\begin{array}{c}\text { Personas } \\
\text { ajenas al } \\
\text { hogar }\end{array}$ & Nadie & Nc \\
\hline Ropa & 1 & 2 & 3 & 4 & 5 & 9 \\
\hline Cocinar & 1 & 2 & 3 & 4 & 5 & 9 \\
\hline Limpieza & 1 & 2 & 3 & 4 & 5 & 9 \\
\hline $\begin{array}{l}\text { Dedicación niños dentro del } \\
\text { hogar }\end{array}$ & 1 & 2 & 3 & 4 & 5 & 9 \\
\hline Actividades extraescolares & 1 & 2 & 3 & 4 & 5 & 9 \\
\hline Cuidados médicos & 1 & 2 & 3 & 4 & 5 & 9 \\
\hline
\end{tabular}


Construcción y validación de un instrumento para la medición de dimensiones sociales en las prácticas...

\section{Subescala conciliación entrenamiento larga distancia con tareas blandas}

-En una semana normal de entrenamiento de larga distancia, EN TU HOGAR/ EN CUANTO A LA ATENCIÓN DE LOS HIJOS ¿quién realiza las siguientes tareas principalmente? (mover la barra hacia la derecha para ver todas las opciones)

\begin{tabular}{lcccccc}
\cline { 2 - 7 } & & & \multicolumn{3}{c}{ Personas } \\
& Yo & Mi pareja & $\begin{array}{c}\text { De forma } \\
\text { compartida }\end{array}$ & $\begin{array}{c}\text { hogar } \\
\text { hogar }\end{array}$ & Nadie & Nc \\
\hline Compra & 1 & 2 & 3 & 4 & 5 & 9 \\
\hline Pequeñas reparaciones (bricolaje) & 1 & 2 & 3 & 4 & 5 & 5 \\
\hline Dedicación niños fuera del hogar & 1 & 2 & 3 & 4 & 5 & 9 \\
\hline Gestiones (papeleos) & 1 & 2 & 3 & 4 & 5
\end{tabular}

FACTOR: Estatus generado por logros.

Subescala aceptación social

-De los grupos de referencia a los que perteneces, ¿podrías señalar la ACEPTACIÓN que tienen ante el hecho de que hagas larga distancia?

\begin{tabular}{ccccccc}
\hline No tengo & Prefieren & Lo & Lo & Lo & Lo & NC \\
& que no lo & aceptan & aceptan & aceptan y & aceptan, & \\
haga & pero no & (neutral) & ven como & lo ven & \\
& & les gusta & & algo & como & \\
& & & & positivo & positivo y &
\end{tabular}

\begin{tabular}{llllllll}
\hline Padres & 1 & 2 & 3 & 4 & 5 & 6 & 9 \\
\hline Hermanos/as & 1 & 2 & 3 & 4 & 5 & 6 & 9 \\
\hline Pareja & 1 & 2 & 3 & 4 & 5 & 6 & 9 \\
\hline $\begin{array}{l}\text { Compañeros/as } \\
\text { de trabajo/clase }\end{array}$ & 1 & 2 & 3 & 4 & 5 & 6 & 9 \\
\hline Amigos/as & 1 & 2 & 3 & 4 & 5 & 6 & 9 \\
\hline
\end{tabular}

Subescala nivel de satisfacción resultados.

\begin{tabular}{|l|c|c|c|c|c|c|c|c|c|c|c|}
\cline { 2 - 9 } \multicolumn{1}{c|}{} & $\begin{array}{l}\text { Nada satisfecho/a } \\
\text { satisfecho/a }\end{array}$ \\
\begin{tabular}{|l|c|c|c|c|c|c|c|} 
Nivel de satisfacción con tu \\
mejor marca conseguida en \\
distancia medio Ironman
\end{tabular} & 0 & 1 & 2 & 3 & 4 & 5 & 6 & 7 & 8 & 9 & 10 \\
\hline $\begin{array}{l}\text { Nivel de satisfacción con tu } \\
\text { mejor marca conseguida en } \\
\text { distancia Ironman }\end{array}$ & 0 & 1 & 2 & 3 & 4 & 5 & 6 & 7 & 8 & 9 & 10 \\
\hline
\end{tabular}

-Nivel de satisfacción de RESULTADOS. ¿Cuál es tu nivel de satisfacción con los resultados obtenidos a nivel global? (debes valorar si estás satisfecho con el los resultados globales obtenidos)

\begin{tabular}{cccccc}
\hline $\begin{array}{c}\text { Nada } \\
\text { satisfecho }\end{array}$ & Poco satisfecho & Término medio & $\begin{array}{c}\text { Bastante } \\
\text { satisfecho }\end{array}$ & $\begin{array}{c}\text { Muy } \\
\text { satisfecho }\end{array}$ & NC \\
\hline 1 & 2 & 3 & 4 & 5 & 9 \\
\hline
\end{tabular}


ELOY GARCÍA GARCÍA es licenciado en Sociología por la UA, licenciado en Investigación y técnicas de mercado por la $\mathrm{UMH}$, doctorando en la UA en el programa Estudios Interdisciplinares de Género, educador y profesor en el departamento de Sociología II de la UA. Su principal línea de investigación es la sociología del deporte.

Recibido: $02 / 11 / 2016$

Aceptado: 30/11/2016 